\title{
Obtenção de nanotubos de óxido de cério através de síntese hidrotérmica alcalina livre de templates
}

\author{
Adriana P. B. Santos¹; Anne Gabriella D. Santos²; J osé Alzamir P. Costa²; Luiz D. Souza²; Antonio S. \\ Araújo $^{1}$ \\ 'Universidade Federal do Rio Grande do Norte, Laboratório de Catálise e Petroquímica, Instituto de \\ Química, 59078-970, Natal RN. *adriana_pbs@yahoo.com.br \\ 2Universidade do Estado do Rio Grande do Norte, Laboratório de Catálise, Ambiente e Materiais, \\ Departamento de Química, 59610-210, Mossoró RN.
}

\begin{abstract}
Resumo:
Nanotubos constituídos de óxidos de metais têm atraído atenção na última década em virtude de suas propriedades físicas e químicas diferentes das do material de origem. Materiais constituídos de cério mostram características ópticas, eletrônicas e magnéticas interessantes, principalmente devido à presença de elétrons na camada $\mathrm{f}$. Na literatura, as metodologias mais comumente utilizadas para a obtenção de nanotubos de Cério (CeNTs) utilizam templates como direcionadores de estrutura. Este trabalho buscou obter CeNTs por meio de síntese hidrotérmica alcalina, sem a utilização de templates. O material obtido foi caracterizado por TG-DSC, DRX, MEV e Adsorção/Dessorção de N2. Os resultados mostraram a obtenção de um material nanotubular constituído inicialmente de $\mathrm{Ce}(\mathrm{OH})_{3}$ visualizado pelas curvas de TG-DSC. Os difratogramas exibiram um aumento de organização estrutural após a calcinação do nanotubo de $\mathrm{Ce}(\mathrm{OH})_{3}$ e obtenção de nanotubos de $\mathrm{CeO}_{2}$ (CeNTs). A morfologia nanotubular foi confirmada pelas imagens de MEV, mostrando materiais em multicamadas com diâmetros externo médio na faixa de $30-90 \mathrm{~nm}$. As isotermas de Adsorção/Dessorção de $\mathrm{N}_{2}$ obtidas para o CeNTs indicou loop de histerese do tipo C, típica de morfologia nanotubular. Os CeNTs obtidos possuem uma área superficial específica de $60,87 \mathrm{~m}^{2} / \mathrm{g}$.
\end{abstract}

\section{Palavras-chave:}

Síntese; hidrotermica; óxido de cério; nanotubos.

Espaço reservado para organização do congresso. 


\section{I ntrodução}

Estruturas nanométicas constituídas de óxidos de metais têm atraído atenção na última década em virtude de suas propriedades físicas e químicas fascinantes e diferentes das do material de origem (NEFZI e SEDIRI, 2013). Esforços têm sido destinados na obtenção e compreensão de suas nanoestruturas unidimensionais (1D), que podem apresentar propriedades elétricas, ópticas, magnéticas, mecânicas e térmicas únicas (PLATA et al, 2012). Estes materiais nanotubulares mostram aplicações em muitas áreas, como em catálise, na liberação de fármacos e na proteção de espécies biológicas sensíveis presentes no meio ambiente (CHEN et al, 2009).

De acordo com a literatura (NADIMICHERLA et al, 2014), o método mais eficaz para a obtenção de materiais nanotubulares ou ainda de outras nanostruturas 1-D é a síntese hidrotérmica, já que exige uma baixa temperatura para a obtenção de uma grande escala, o que torna o processo mais barato, podendo ainda ser aplicado a vários tipos de nanomateriais.

Em 1998, Kasuga e colaboradores (KASUGA et al, 1998) mostraram a possibilidade de obtenção de nanotubos de óxido de titânio através de uma síntese simples utilizando tratamento hidrotérmico alcalino. Este trabalho mostrou a possibilidade de conversão completa do material de partida $\left(\mathrm{TiO}_{2}\right.$ anatásio) em estruturas nanotubulares. De acordo com a literatura (MORGADO et al, 2007), a formação dos nanotubos por essa rota ocorre durante a reação hidrotérmica alcalina. Inicialmente ocorre a quebra de ligações químicas da estrutura dimensional do óxido metálico, formando-se estruturas planares (2-D), as quais são dobradas gerando os nanotubos.

Os materiais constituídos de cério, assim como todas as terras raras, mostram características ópticas, eletrônicas e magnéticas principalmente devido à presença de elétrons na camada $\mathrm{f}$ (KUANG et al, 2007). O Ce na forma de óxido é considerado um dos materiais mais reativos dos óxidos de terras raras, refletindo na sua extensiva aplicações, sendo elas em catálise heterogênea, luminecência, células de combustíveis, células solares (em virtude de sua estabilidade química), alta capacidade de armazenamento de oxigênio, catalisadores bloqueadores de UV e em materiais de polimento (CHEN et al, 2009; PLATA et al, 2012). E é em virtude de suas novas propriedades estruturais e aplicações potenciais que os nanotubos de $\mathrm{CeO}_{2}$ tem sido bastante investigados.

Vários são os métodos utilizados para a obtenção destes materiais nanotubulares, utilizando como template a alumina porosa ou ainda nanotubos de carbonos. Pan et al., (2008), mostrou a utilização de CTABr como molde para formação destes nanotubos. Chen et al., (2010), utilizou attapugite (silicato lamelar de magnésio e alumínio) como template. Os resultados mostraram que o diâmetro resultante dos nanotubos obtidos se mostraram semelhantes aos moldes utilizados nas sínteses. As demais desvantagens para estas rotas que utilizam template é a necessidade da sua remoção, o que torna o processo mais caro. Além disso, como a dimensão do nanotubo é governada pelo molde, mudanças nas condições de reação quase não promoveriam ajustes de interesse no material final (WU e KAWI 2010). A não utilização de templates para a obtenção destes nanomateriais mostra ainda como vantagens o fato de como estes direcionadores de estruturas serem constituídos de materiais orgânicos podem gerar diversos problemas quando utilizados, como manuseio de produtos tóxicos e corrosivos, contaminação de recursos hídricos e terrestres, poluição atmosférica (calcinação) e obstrução de poros do nanotubo com a formação de coque em virtude da combustão incompleta durante o processo de calcinação para a remoção destes templates (CALDEIRA, 2011).

$\mathrm{Na}$ literatura, podemos encontrar algumas sínteses de nanotubos de $\mathrm{CeO}_{2}$ livres de templates. Han et al., (2005) obteve nanotubos de $\mathrm{CeO}_{2-x}$ por precipitação e envelhecimento, utilizando nitrato de cério e rota hidrotérmica, com tempo de envelhecimento de 45 dias, tempo desfavorável para produção em larga escala. Tang et al., (2005) sintetizaram nanotubos de $\mathrm{CeO}_{2}$ submetendo a tratamento hidrotérmico alcalino o cloreto de lantânio, obtendo um pó 
de coloração roxa. Chen et al., (2009) fabricou nanotubos de $\mathrm{CeO}_{2}$ através da reação de interface sólido-líquido entre nanobastões de $\mathrm{Ce}(\mathrm{OH}) \mathrm{CO}_{3}$ e solução de hidróxido de sódio.

Neste trabalho, nanotubos de cério (CeNTs) foram sintetizados por meio de síntese hidrotérmica alcalina livre de templates, utilizando uma temperatura de envelhecimento de $125{ }^{\circ} \mathrm{C}$ e tempo de síntese de $72 \mathrm{~h}$. Os materiais obtidos foram caracterizados por TG-DSC, DRX, MEV e adsorção/dessorção de $\mathrm{N}_{2}$ a fim de se obter uma melhor compreensão das estruturas obtidas.

\section{Metodologia}

\subsection{Síntese dos CeNTs}

Os $\mathrm{Ce}(\mathrm{OH})_{3}-\mathrm{NTs}$ foram obtidos através de síntese hidrotérmica alcalina sob condições livres de oxigênio. Para isto, a solução de $\mathrm{NaOH}(11 \mathrm{M})$ foi submetida a ultrason por 20 min para a remoção do $\mathrm{O}_{2}$. Em um teflon revestido de aço inoxidável (autoclave), com capacidade de $40 \mathrm{~mL}$, foi adicionado $\mathrm{Ce}\left(\mathrm{NO}_{3}\right)_{3} 6 \mathrm{H}_{2} \mathrm{O}$ e posteriormente a solução de $\mathrm{NaOH}$ livre de $\mathrm{O}_{2}$. A autoclave foi bem fechado e submetido a aquecimento em estufa a $125^{\circ} \mathrm{C}$ por $72 \mathrm{~h}$. Após o tratamento hidrotérmico, o $\mathrm{Ce}(\mathrm{OH})_{3^{-}}$ NTs obtido foi lavado com água deionizada livre de $\mathrm{O}_{2}$ até pH neutro e seco em estufa a $100{ }^{\circ} \mathrm{C}$ por 10 h. A obtenção do CeNTs ocorreu através de calcinação em forno mufla do $\mathrm{Ce}(\mathrm{OH})_{3}$-NTs a $450{ }^{\circ} \mathrm{C}$ por $5 \mathrm{~h}$, com rampa de aquecimento de $10^{\circ} \mathrm{C} / \mathrm{min}$.

\subsection{Caracterização dos CeNTs}

As curvas termogravimétricas e calorimétricas (TG/DSC) foram realizadas em equipamento da Netzsch, modelo STA 449 F3 Jupiter. Foram utilizadas aproximadamente 5,0 mg de amostra em cadinhos de alumina que foram aquecidos até $900{ }^{\circ} \mathrm{C}$ com razão de aquecimento de $10{ }^{\circ} \mathrm{C} / \mathrm{min}$ em atmosfera inerte de $\mathrm{N}_{2}$ com fluxo de $60 \mathrm{~mL} / \mathrm{min}$. As curvas de TG informaram a temperatura necessária para a obtenção de nanotubos de $\mathrm{CeO}_{2}$, a partir do $\mathrm{Ce}(\mathrm{OH})_{3}$-NTs. Os dados de Difração de Raios-X (DRX) do pó foi coletado em equipamento da Rigaku modelo Mini Flex II, usando radiação $\mathrm{K} \alpha$ de $\mathrm{Cu}\left(\lambda=1.5406^{\circ} \mathrm{A}\right)$. As medidas foram realizadas pelo método de varredura, de $2 \theta$ de 1 a $90^{\circ}$, passo de 0,02 graus/s e tempo de 1s. As imagens de MEV dos nanotubos obtidos foram analisados em Microscópio Eletrônico de Varredura (MEV) da TESCAN MIRA 3, em faixa de magnificação de 6000X a 16000X. As medidas de adsorção/dessorção de $\mathrm{N}_{2}$ foram obtidas por meio de equipamento ASAP 2020 (Micromeritics). Anteriormente a análise, as amostras foram desgaseificadas a $300{ }^{\circ} \mathrm{C}$ por 10 horas. Foi utilizada a equação de Brunauer-Emmet-Teller (BET) para determinar a área superficial específica e o método Barrett-Joyner-Halenda (BJH) foi utilizado para calcular a distribuição do tamanho de poro.

\section{Resultados e Discussões}

O Gráfico 1 mostra as curvas de TG-DSC do material obtido na síntese. Esta análise objetivou avaliar a composição e estabilidade dos nanotubo obtido. As curvas de TG mostram quatro eventos com variações de massa, que se inicia em $25^{\circ} \mathrm{C}$ e finaliza em torno de $900^{\circ} \mathrm{C}$. A saída de água adsorvida no material ocorre em I (9,54\%), iniciando $25{ }^{\circ} \mathrm{C}$ finalizando em $120^{\circ} \mathrm{C}$, (Andreescu et al., 2006). A segunda perda de massa, evento II (32,6\%), que ocorre entre 120 e $450{ }^{\circ} \mathrm{C}$, sendo atribuído ao processo de desidratação do diâmetro externos dos $\mathrm{Ce}(\mathrm{OH})_{3}$-NTs. O evento III (15,09 \%) é observado na faixa de 450 a $600{ }^{\circ} \mathrm{C}$,e tem a ver com a desidratação do diâmetro internos dos $\mathrm{Ce}(\mathrm{OH})_{3}$-NTs. Estas perdas de massas observadas sugerem que o material sintetizado consiste de óxido de cério hidratado (Wu e Kawi 2010). Um quarto evento de ganho de massa pode ser observado na faixa de 600 a 900 ${ }^{\circ} \mathrm{C}$. De acordo com Santos et al, 2015, isto ocorre em virtude do colapso das camadas dos nanotubos pela oxidação do cério de $\mathrm{Ce}^{2+}$ para $\mathrm{Ce}^{3+}$, ao mesmo tempo que ocorre a conversão para cristalitos de óxido de cério. A curva de DSC mostra um pico endotérmico para os três primeiros eventos e um vento exotérmico incompleto para o quarto evento, correspondente ao colapso das nanoestruturas e oxidação do material. 


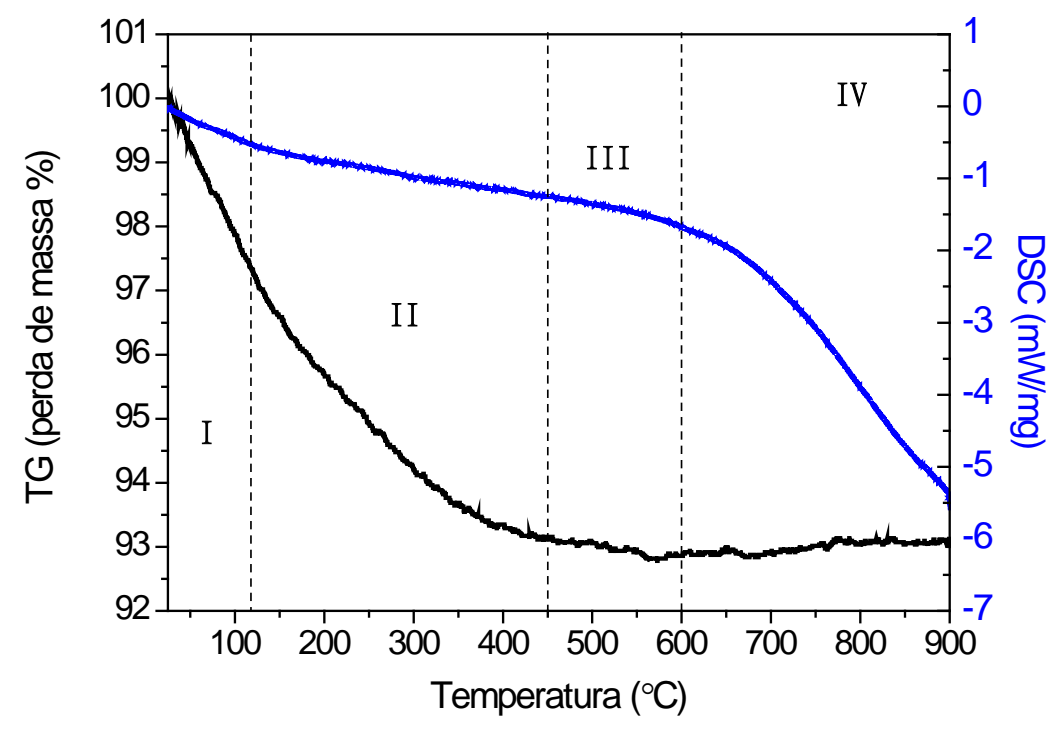

Gráfico 1. Curvas TG-DSC dos $\mathrm{Ce}(\mathrm{OH})_{3}-\mathrm{NTs}$ obtidos na síntese.

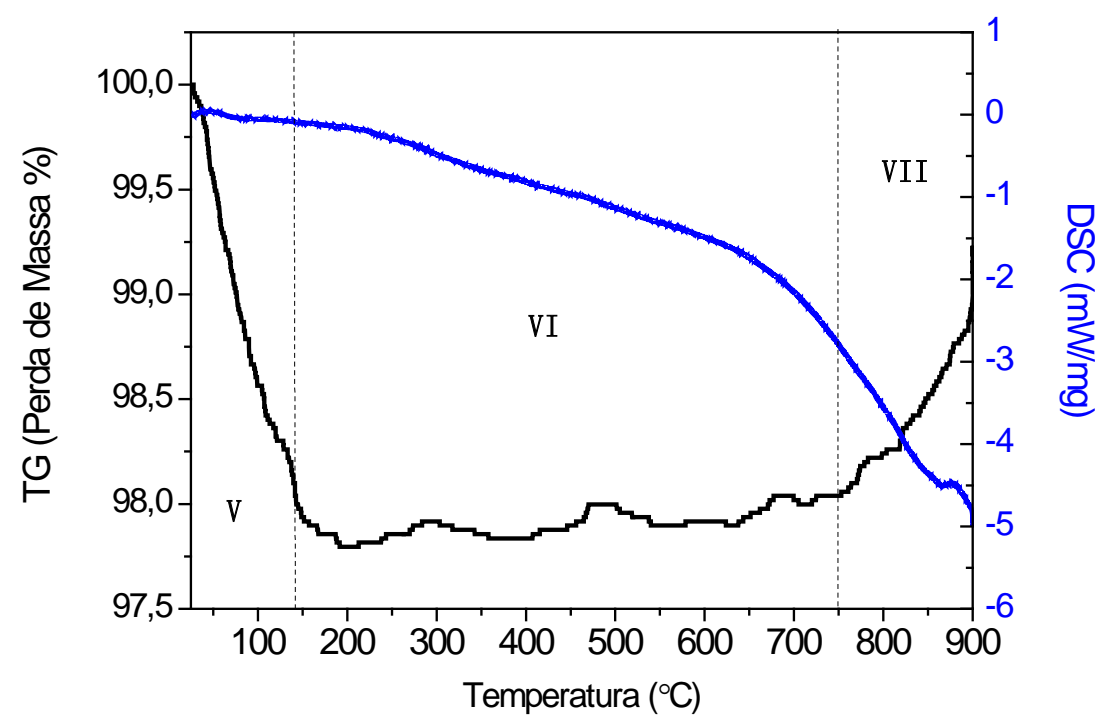

Gráfico 2. Curvas TG-DSC dos CeNTs obtidos após a calcinação.

No Gráfico 2 está exposto as curvas de TG/DTG do CeNTs obtido após o processo de calcinação. Podemos observar que o material mostra ainda perda de massa até e torno de $140{ }^{\circ} \mathrm{C}$, referente a saída de água ligada fisicamente ao material. Em seguida, é perceptível oscilações de ganho e perda de massa, que pode ser atribuído aos ruídos inerentes a este tipo de análise. Em temperaturas acima de $750{ }^{\circ} \mathrm{C}$ observa-se mais uma vez um ganho de massa atribuído à oxidação e colapso das estruturas nanotubulares. Contudo, estes resultados mostram que o processo de calcinação foi eficiente para remover toda a água de hidratação presente nos $\mathrm{Ce}(\mathrm{OH})_{3}$-NTs. Detalhes de todas as variações de massas envolvidas para os Gráficos 1 e 2 podem ser vistas na Tabela 1. 
Tabela - 1 Perdas de massas envolvidas nos eventos térmicos para o material sintetizado.

\begin{tabular}{ccc}
\hline Evento térmico & Faixa de temperatura $\left({ }^{\mathbf{0}} \mathbf{C}\right)$ & Variações de Massa (\%) \\
\hline I & $25-120$ & $-9,53$ \\
\hline I I & $120-450$ & $-32,60$ \\
\hline III & $450-600$ & $-15,09$ \\
\hline IV & $600-900$ & $+0,24$ \\
\hline V & $25-140$ & $-1,96$ \\
\hline VI & $140-750$ & 0 \\
\hline VII & $750-900$ & $+0,91$ \\
\hline
\end{tabular}

O Gráfico 3 mostra os difratogramas de raios-X do material obtido na síntese $\left(\mathrm{Ce}(\mathrm{OH})_{3}-\mathrm{NTs}\right)$ e do material após a calcinação (CeNTs). Podemos observar a presença dos mesmos picos de reflexão nos dois difratogramas, em $2 \theta: 28,42^{\circ}, 32,97^{\circ}, 47,42^{\circ}$ e $56,23^{\circ}, 58,84^{\circ}, 68,86^{\circ}, 76,2^{\circ}, 78,46^{\circ}$, e $87,56^{\circ}$. Estes picos, assim como descreve Wu e Kawi (2010), são típicos da presença de nanotubos de $\mathrm{Ce}(\mathrm{OH})_{3}$, como também da presença de nanotubos de $\mathrm{CeO}_{2}$ (Cho et al., 2015). De acordo com PDFno.:43-0394, estes picos de reflexão correspondem ao $\mathrm{CeO}_{2}$ com estrutura na forma de fluorita. É perceptível ver que após o processo de calcinação, ocorre um aumento de intensidade dos referidos picos, o que indica um aumento na organização nanotubular.

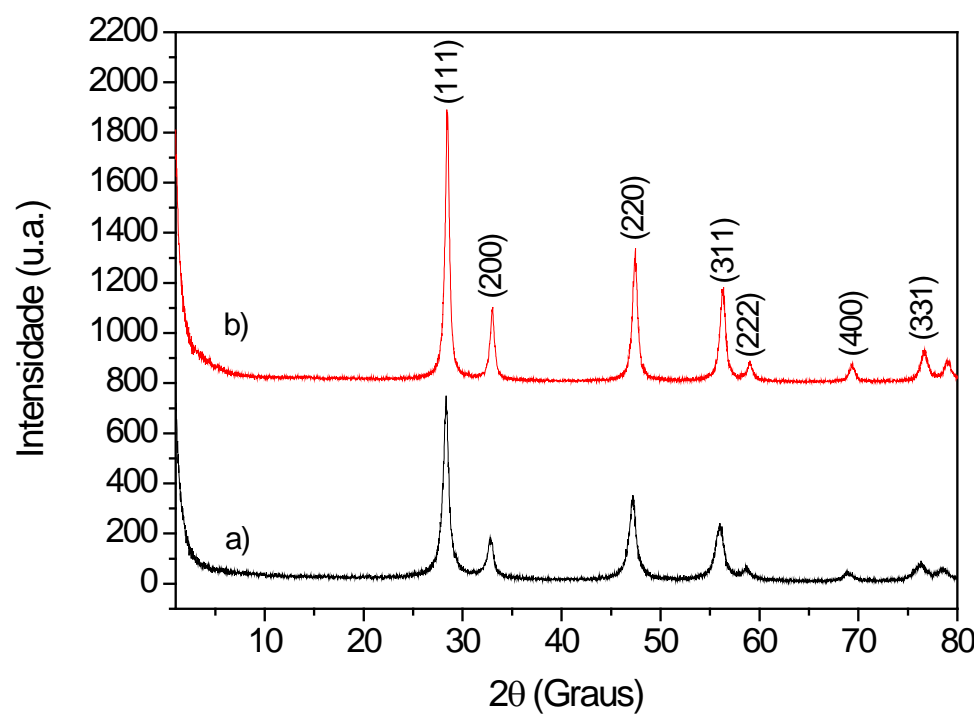

Gráfico 3. DRX do (a) $\mathrm{Ce}(\mathrm{OH})_{3}$-NTs e (b) do CeNTs obtidos após a calcinação.

A micrografia do material sintetizado pode ser visto na Figura 1, com a ampliação de um nanotubo em $a$ A imagem mostra claramente a formação nanotubular do material, com estrutura unidirecional. É perceptível também observar que a formação nanotubular ocorreu com um alto rendimento. A ampliação em $a$, permite-nos ver a formação nanotubular em multicamadas. As nanoestruturas obtidas mostraram diâmetro externo médio na faixa de $30-90 \mathrm{~nm}$, valores próximos do mostrado na literatura (Wu e Kawi 2010; Tang et al., 2005). Somente por meio da análise por MET é possível estimar o diâmetro interno dos nanotubos sintetizados.

As isotermas de adsorção/dessorção dos nanotubos calcinados podem ser vistas na Figura 1. É possível verificar a presença de isotermas do tipo IV com loop de histerese na faixa de 0,8-1,0, indicando material microporoso na forma nanotular (Wu et al 2015; Chen et al 2014). O tipo de loop 
de histerese de materiais nanotubulares é o $\mathrm{C}$, em virtude da presença de mistura de nanotubos na forma cônica ou em forma de cunhas abertas (Pan et l 2008). O CeNTs obtido uma interessante área superficial específica (BET) de $60,87 \mathrm{~m}^{2} / \mathrm{g}$ e distribuição do tamanho de poro em torno de 16,5 $\mathrm{nm}$.

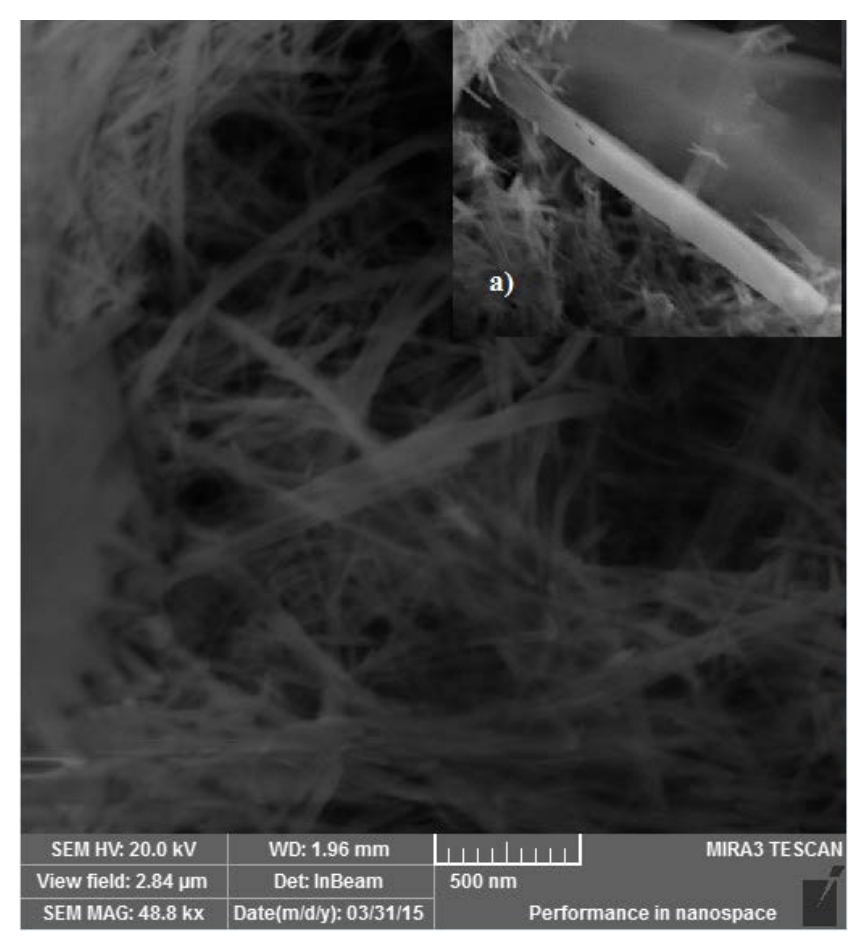

Figura 1. Imagem de $\mathrm{MEV}$ do $\mathrm{Ce}(\mathrm{OH})_{3}$-NTs.

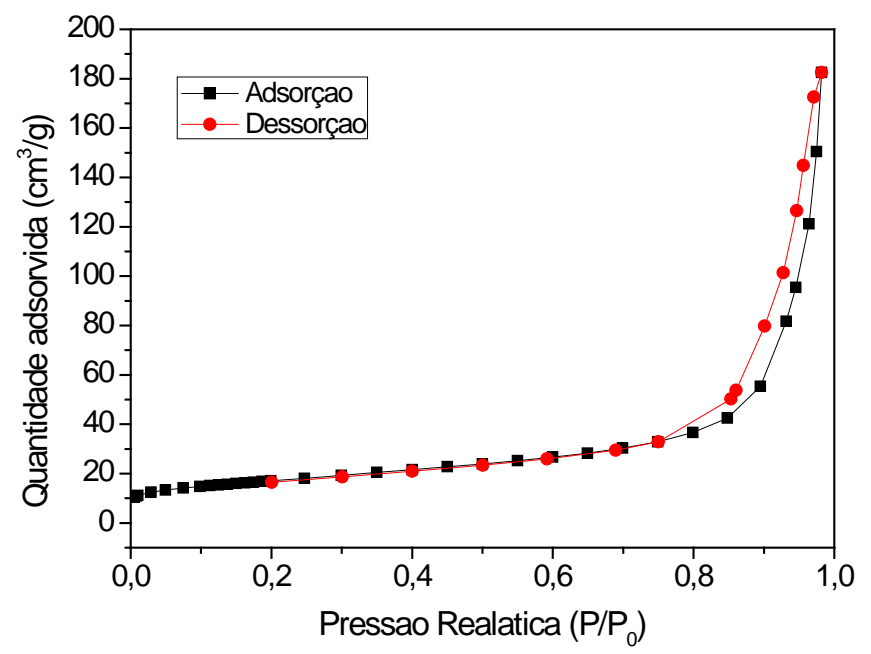

Gráfico 4. Isotermas de adsorção/dessorção de $\mathrm{N}_{2}$ para o CeNTs obtidos.

A curva de distribuição do tamanho de poro, que pode ser vista na Gráfico 5, propõe que o tamanho dos poros dos nanotubos está principalmente centrado entre $10-27 \mathrm{~nm}$ e 34 a $75 \mathrm{~nm}$. 


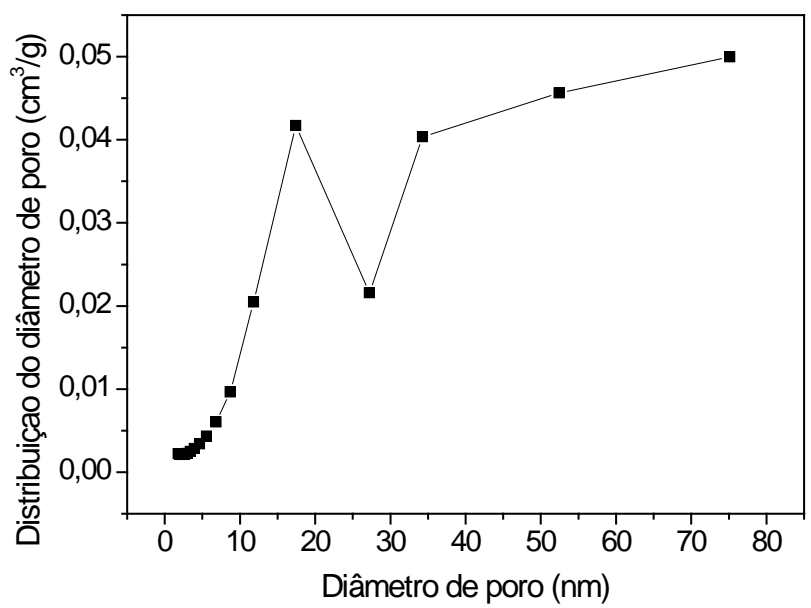

Gráfico 5. Distribuição do tamanho de poro dos CeNTs obtidos.

De acordo com Wu e Kawi (2010), a síntese utilizando nitrato de cério e solução de hidróxido de sódio concentrada baseia-se no mecanismo de dissolução e recristalização do $\mathrm{Ce}(\mathrm{OH})_{3}$, onde ocorre o crescimento gradual do $\mathrm{Ce}(\mathrm{OH})_{3}-\mathrm{NTs}$, ao longo do eixo c. Na figura 2 abaixo esquematiza um tipo de mecanismo de formação dos nanotubos mencionados, através do crescimento vertical dos nanotubos sobre uma base inicial plana:

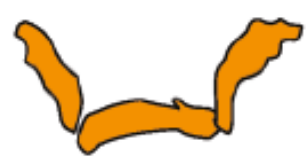

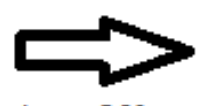

Após 12h

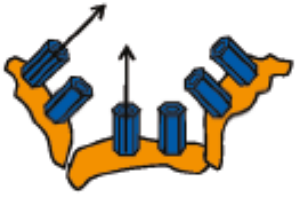

Recristalização e crescimento vertical

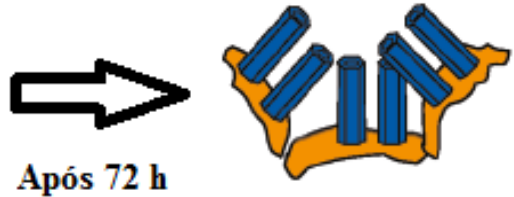

Após 72 h

Figura 2. Ilustração do crescimento vertical de $\mathrm{Ce}(\mathrm{OH})_{3}-\mathrm{NTs}$ sobre uma base plana (Adaptado de Wu e Kawi, 2010).

Um outro tipo possível de mecanismo para a formação dos nanotubos de $\mathrm{Ce}(\mathrm{OH})_{3}-\mathrm{NTs}$, baseado no crescimento multidirecional destes nanotubos sobre uma base de núcleo esférico, como ilustrado na Figura 3a seguir.
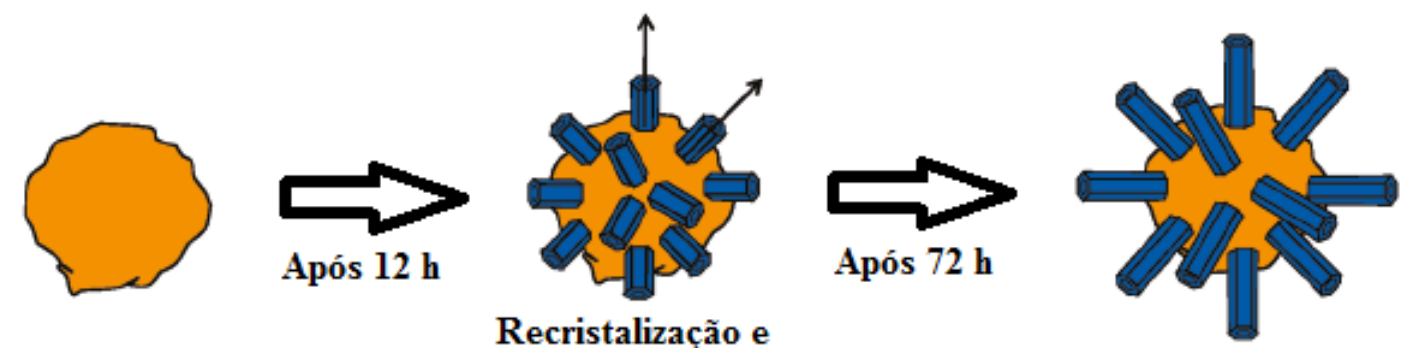

Recristalização e crescimento multidirecinal

Figura 3. Ilustração do crescimento multi-direcional sobre uma base esférica (Adaptado de Wu e Kawi, 2010).

\section{Conclusões}


Neste estudo, os nanotubos de $\mathrm{CeO}_{2}$ foram obtidos com sucesso pelo método hidrotémico alcalino livre de templates, através de um período de envelhecimento de $72 \mathrm{~h}$. O material nanotubular obtido inicialmente foi o $\mathrm{Ce}(\mathrm{OH})_{3}$-NTs, confirmado pelas curvas de TG/DSC. Onde após o processo de calcinação foram sendo transformado em nanotubos de $\mathrm{CeO}_{2}$, adquirindo assim uma melhor organização, vista pelos difratogramas. A morfologia nanotubular foi comprovadas pelas imagens de MEV, bem como sua formação em multicamadas. As isotermas de Adsorção/Dessorção de $\mathrm{N}_{2}$ exibiram perfil típico de estruturas nanotubulares, com nanotubos com interessantes áreas superficiais específica e distribuição de tamanho de poro. Como o esperado, foi obtido com alto rendimento de um material com morfologia nanotubular, baseado em um mecanismo de dissolução e recristalização de formação.

\section{Obtaining cerium oxide nanotube by template-free alkaline hydrothermal synthesis}

Albstract: Nanotubes made of metal oxides have attracted attention in the last decade due to their physical and chemical properties different from the parent material. Consisting of cerium materials show optical characteristics, electronic and magnetic interesting, mainly due to the presence of electrons in the f layer. In literature, the methods most commonly used for obtaining cerium nanotubes (CeNTs) using template as structure-directing.This study aimed to obtain cerium oxide nanotubes (CeNTs) by alkaline hydrothermal synthesis without the use of templates. The material obtained was characterized by TG-DSC, XRD, SEM and $\mathrm{N}_{2}$ adsorption/desorption. The results showed obtaining a nanotubular materials initially consisting of $\mathrm{Ce}(\mathrm{OH})_{3}$ displayed by the TG-DSC curves. The XRD patterns showed an increase in structural organization of the calcining nanotube after $\mathrm{Ce}(\mathrm{OH})_{3}$ to obtain $\mathrm{CeO}_{2}$ nanotubes. The nanotubular morphology was confirmed by SEM images showing multilayer materials with an average external diameter in the range 30-90 $\mu \mathrm{m}$. The isotherms adsorption/desorption of $\mathrm{N}_{2}$ obtained for CeNTs hysteresis loop indicated type C typical nanotubular morphology. The CeNTs obtained have a specific surface area of $60.87 \mathrm{m2} / \mathrm{g}$.

Keywords: synthesis; hydrothermal; cerium oxide; nanotubes.

\section{Referências bibliográficas}

ANDREESCU, Daniel; MATIJ EVI 'C, Egon; GOIA, Dan V. Formation of uniform colloidal ceria in polyol. Colloids and Surfaces A: Physicochem. Engineering Aspects, 291, 93-100, 2006.

CALDEIRA, Vinicius. P., Dissertação de Mestrado, PPGQ/UFRN, Natal-RN, 2011.

CHEN, Guozhu; SUN, Sixiu; SUN, Xun; FAN, Weiliu; YOU, Ting. Formation of CeO2 Nanotubes from $\mathrm{Ce}(\mathrm{OH}) \mathrm{CO}_{3}$ Nanorods through Kirkendall Diffusion. I norganic Chemistry, 48, 1334-1338, 2009.

CHEN, Zhigang; CHEN, Feng; LI, Xiazhang; LU, Xiaowang; NI, ZHAO Xiaobing. Facile synthesis of $\mathrm{CeO}_{2}$ nanotubes templated by modified attapulgite. Journal of Rare Earths, 28, 566-570, 2010.

CHEN, Zhonghui; ZHANG, Jianan; FU, Jianwei, WANG, Minghuan; WANG, Xuzhe; HANB, Runping; XU, Qun. Adsorption of methylene blue ontopoly(cyclotriphosphazene-co-4,4_-sulfonyldiphenol) nanotubes:Kinetics, isotherm and thermodynamics analysis. Journal of Hazardous Materials. 273, 263-271,2014. 
CHO, Young Jun; JANG, Hanmin; LEE, Kwan-Soo; KIM, Dong Rip. Direct growth of cerium oxide nanorods on diverse substrates forsuperhydrophobicity and corrosion resistance. Applied Surface Science, 340, 96-101, 2015.

HAN, Wei-Qiang; WU, Lijun, ZHU, Yimei. Formation and Oxidation State of CeO2-x Nanotubes. J ounal of American Chemical Society, 127, 12814-12815, 2005.

KASUGA, Tomoko; HIRAMATSU, Masayoshi; HOSON, Akihiko; SEKINO, Toru; NIIHARA, Koichi. Formation of Titanium Oxide Nanotube. Langmuir, 14, 3160-3163, 1998.

KUANG, Qin.; LIN, Zhi-Wei.; LIAN, Wei.; JIANG, Zhi-Yuan.; XIE, Zhao-Xiong.; HUANG, Rong-Bin.; ZHENG, Lan-Sun. Syntheses of rare-earth metal oxide nanotubes by the sol-gel methodassisted with porous anodic aluminum oxide templates. J ournal of Solid State Chemistry, 180, 1236-1242, 2007.

MORGADO Jr. Edisson; ABREU, Marcos; MOURE, Gustavo; MARINKOVIC, Bojan; JARDIM, Paula; ARAUJ O, Araujo. Effects of thermal treatment of nanostructured trititanates on their crystallographic and textural properties. Materials Research Bulletin, 42, 1748-1760, 2007.

NADI MI CHERLA, Reddeppa; LIU, Yueli; CHEN, Keqiang; CHEN, Wen Chen. Effect of polyethylene glycol on vanadium oxide nanotubes in lithium-ion batteries. Microelectron Engineering, 127, 8185, 2014.

NEFZ, H; SEDIRI, F. Vanadium oxide nanotubes VOx-NTs: Hydrothermal synthesis, characterization, electrical study and dielectric properties. J ournal of Solid State Chemistry, 201, 237-243, 2013.

PAN, Chengsi; ZHANG, Dengsong; SH, Liyi. CTAB assisted hydrothermal synthesis, controlled conversion and $\mathrm{CO}$ oxidation properties of $\mathrm{CeO} 2$ nanoplates, nanotubes, and nanorods. Journal of Solid State Chemistry, 181, 1298, 2008.

PLATA, J osé J .; RUIZ-TAGLE, Igor; MÁRQUEZ, Antonio M.; SANZ, Javier Fdez. Ceria(100) Nanotubes with Negative Strain Energy: A First-Principles Prediction. The Journal of Physical Chemistry Letters, 3, 2092-2096, 2012.

SANTOS, Adriana; FERNANDES, Rodrigo; SANTOS, Anne Gabriela; CALDEIRA, Vinicius; SOUZA, Luiz; ARAÚJ O, Antônio. Nanotubos de óxido de vanádio obtidos com diferentes temperaturas de tratamento hidrotérmico sem o uso de templates orgânicos. 18을 Congresso Brasileiro de Catálise. Arraia D'Ajuda, Porto Seguro, Bahia,2015.

TANG, Chengchun; BANDO, Yoshio; LIU, Baodan; GOLBERG, Dmitri. Cerium Oxide Nanotubes Prepared from Cerium Hydroxide Nanotubes. Advanced Materoals, 17, 3005-3009, 2005.

WU, Xusheng; KAWI, Sibudjing. Synthesis, Growth Mechanism, and Properties of Open-Hexagonal and Nanoporous-Wall Ceria Nanotubes Fabricated via Alkaline Hydrothermal Route. Crystal Growth \& Design, 10, 1833-1841, 2010.

WU, Zhi-Liang; YANG, Hua; JIAO, Fei-Peng; LIU, Qi; CHEN, Xiao-Qing; YU, Jin-Gang. Carbon nanoparticles pillared multi-walled carbon nanotubes foradsorption of 1-naphthol: Thermodynamics, kinetics and isotherms. Colloids and Surfaces A: Physicochem. Engineering Aspects 470, 149-160, 2015. 